\title{
MEASURING CHANGES IN MESOLIMBIC DOPAMINE AND RELATED SUBSTANCES IN RESPONSE TO SYSTEMIC NICOTINE USING IN VIVO ELECTROCHEMISTRY
}

\author{
Graham C. Parker and Paul B.S. Clarke \\ Dept. of Pharmacology \& Therapeutics, McGill University, Montreal, Canada H3G 1Y6
}

\begin{abstract}
Summary: Several psychopharmacological properties of tobacco smoking are believed to be mediated by central nicotinic receptors on mesolimbic dopamine (DA) neurons. Using in vivo electrochemical probes we monitored the extracellular levels of DA alone and also the levels of DOPAC and ascorbate, in the nucleus accumbens following systemic administration of nicotine $(0.4 \mathrm{mg} / \mathrm{kg} \mathrm{SC})$. These studies used nicotine-naive, unanaesthetized freely-moving rats. Preliminary data suggest an initial $(<20 \mathrm{~min})$ decrease in accumbal DA following the first injection of nicotine. This decrease was not observed on the second injection day when the DA signal reached a higher maximum and had a longer duration of effect.
\end{abstract}

\section{Introduction}

One of the goals of neurobiology is to understand how nervous activity in the brain is related to mental functioning and behaviour. Great advances have been made in investigating such psychological constructs as reinforcement, learning and memory by studying the release of neurotransmitters putatively involved in the neurochemistry of such processes. Such behaviours have been demonstrated repeatedly to be affected by manipulations of the catecholamine dopamine (DA) and its release in the nucleus accumbens (NAcc).

Technological advances have allowed us to progress from measuring transmitter levels post mortem to techniques such as microdialysis whereby samples can be extracted for analysis ex vivo from freely moving animals (4). Now we can measure the levels of various neurochemicals in vivo using electrochemistry $(5,6)$. Fortunately, certain monoamines, notably DA and serotonin are electroactive. This means that when a certain voltage is applied across two electrodes, these substances will oxidise, producing a current that can be measured with sensitive equipment. Electroactive substances differ in the voltage at which they will give up 
electrons, hence the identity of the substance can be determined by its oxidative potential, while its concentration local to the electrode surface can be estimated from the size of the resulting current.

\section{Materials and Methods}

Male Long-Evans rats (Charles River, Lachine, Quebec) weighing 300-350 g at time of surgery were housed individually with a $12 \mathrm{hr}$ light/dark cycle (0700/1900 hrs) and ad libitum access to lab chow and water. Under sodium pentobarbital anaesthesia (60 mg / kg IP), working electrodes were implanted bilaterally into the NAcc (level skull, Bregma +1.2 , Mid $\pm 0.6, \mathrm{Ctx}-6.5)$. The working electrodes were constructed using Teflon-coated stainless steel (0.008" bare 0.011 " coated, A-M Systems, Inc.). The Teflon was moved proud of the steel to form a well. The other end of the electrode was soldered to an amphenol pin (Electrosonic). The well was packed with USP grade graphite powder (Ultra Carbon Corp.) that had previously been mixed with $0.8 \mathrm{ml} 0.7 \mathrm{mg} / \mathrm{ml}$ Nujol (Aldrich). Once the well was properly packed the electrode was impacted on a clean flat piece of glass to produce an even compression then expansion of the material producing a spherical electrode surface. The electrode was then dipped in Nafion (in a solution of ethanol/water, see Aldrich Cat no. 27,470-4 for details). The reference electrode comprised Teflon-coated silver wire ( 0.01 " bare, 0.013 " coated) exposed to a length of $1 / 2$ ". The wire was then coiled flat. The other end of the silver wire was soldered to an amphenol connector. The electrode was then chlorided to produce an $\mathrm{Ag} / \mathrm{AgCl}$ reference. It was necessary to carefully drill away the skull above the target coordinates so as to allow a window in the dura to be opened without causing bleeding on the cortex surface. This was to keep the working electrodes as clean as possible when lowered. The implantation of the reference electrode was based on an idea proposed to protect it from deleterious effects of exposure to brain tissue (7). An area of skull 0.01 " in diameter was removed leaving the dura exposed but intact. A circle of gelfoam soaked in $0.9 \% \mathrm{NaCl}$ was placed onto the dura to cover the area. The reference electrode was then lowered onto the gelfoam using a stereotaxic manipulator, another piece of gelfoam placed on top, then covered with dental cement. The auxiliary electrode was a length of stainless steel wrapped round a skull screw implanted so as to be in contact with the dura. By first sweeping through a range of voltages using a potentiostat (E-Chempro Instrument, GMA Computer Technology Ltd.) 
we were able to calculate the voltage required to oxidise DA when we switched to chronoamperometry. Samples were taken every $20 \mathrm{sec}$ without depleting the local extracellular concentration.

Discriminating between various electroactive substances which share a similar oxidative potential is a great challenge in electrochemistry. Ascorbic acid and DOPAC (a metabolite of DA), would swamp any changes in the level of DA observed at a 'plain' electrode because

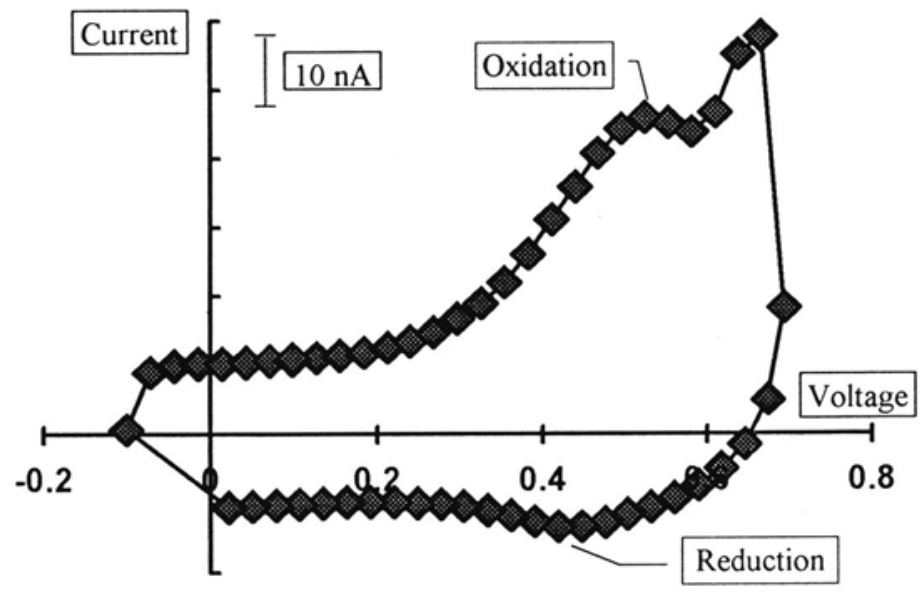

Figure 1. Cyclic voltammogram scanned at $130 \mathrm{mV} / \mathrm{s}$ from a working electrode in the NAcc.

they are present in far greater concentration in the extracellular fluid. To provide the required chemical selectivity, the carbon paste matrix of the working electrode was coated with the perfluorosulfonated polymer Nafion. Since Nafion shares the same charge as ascorbate and DOPAC, it tends to screen these substances out (5), while the oppositely charged DA has access to the electrode surface.

The monoamine oxidase inhibitor, pargyline $(100 \mathrm{mg} / \mathrm{kg}$ IP), blocks the degradation of DA into DOPAC. Hence it is an excellent test for the selectivity of our electrodes. As shown above, an unmodified electrode 'sees' the decrease in the DOPAC signal, while the Nafioncoated electrode only 'sees' the increase in DA. Although the unmodified electrode will also record changes in DA, the higher concentration of DOPAC swamps this signal. Note also the longer time course of effect on DOPAC than DA. Similarly, the potent D1 and D2 agonist pergolide $(0.6 \mathrm{mg} / \mathrm{kg} \mathrm{IP})$, decreases the release of DA while increasing levels of ascorbic acid (8). 


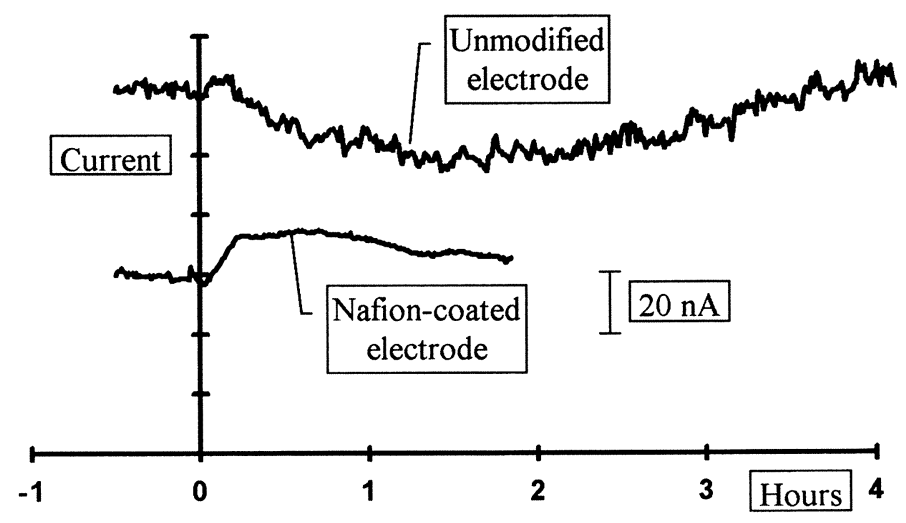

Figure 2. Oxidative current measured in the NAcc following pargyline (100 mg/kg IP).

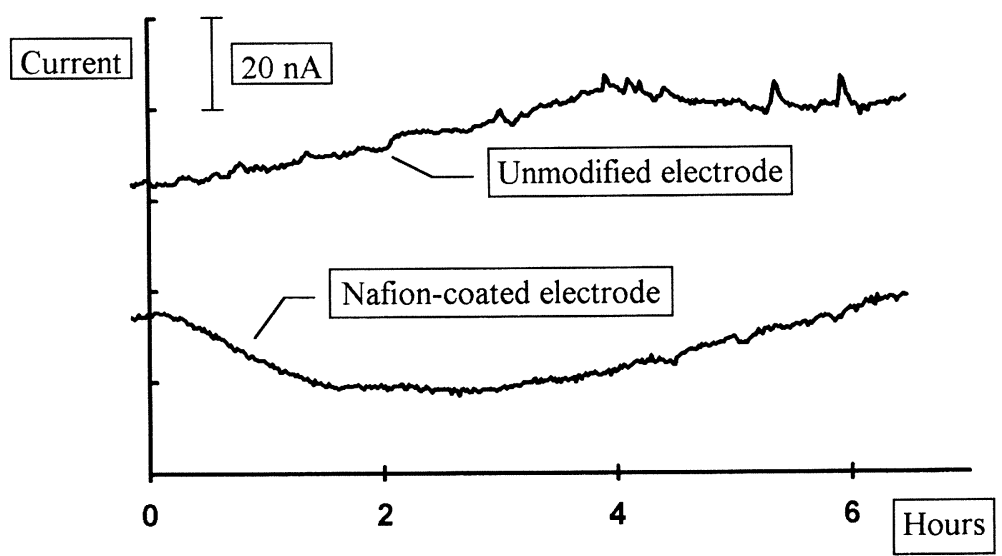

Figure 3. Oxidative current measured in the NAcc following pergolide $(0.6 \mathrm{mg} / \mathrm{kg} \mathrm{IP})$.

\section{Results}

Figure 4 shows a steady increase in the oxidative current, reaching a maximum at $2 \mathrm{hr}$ and not returning to baseline until after $4 \mathrm{hr}$ measured using unmodified electrodes in the NAcc. The increase in current will be produced by increases in DA and/or ascorbic acid, but is probably mostly due to increases in DOPAC. 


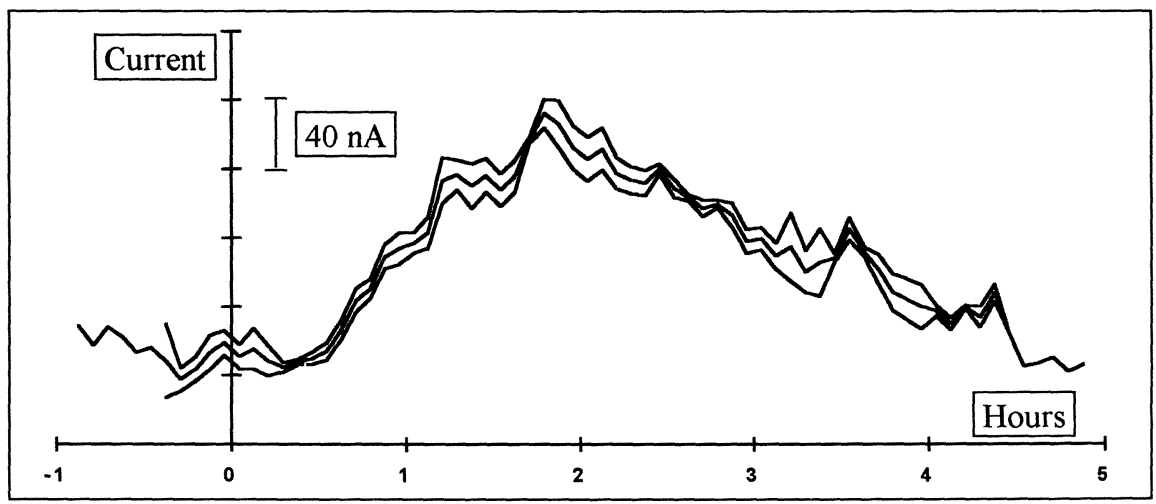

Figure 4. Oxidative current measured at accumbal unmodified electrodes in response to nicotine $(0.4 \mathrm{mg} / \mathrm{kg} \mathrm{SC}$ calculated as base, $\mathrm{pH}$ balanced, $\mathrm{n}=4$ rats, mean $\pm \mathrm{SE})$.

In contrast, the electrodes modified to provide selectivity for DA showed a much shorter duration of effect in the NAcc (Figure 5). On days when a control (saline) injection was given, no response was seen following the injection. However, following the first injection of nicotine there was an initial decrease in the signal which recovered after $15 \mathrm{~min}$, followed by

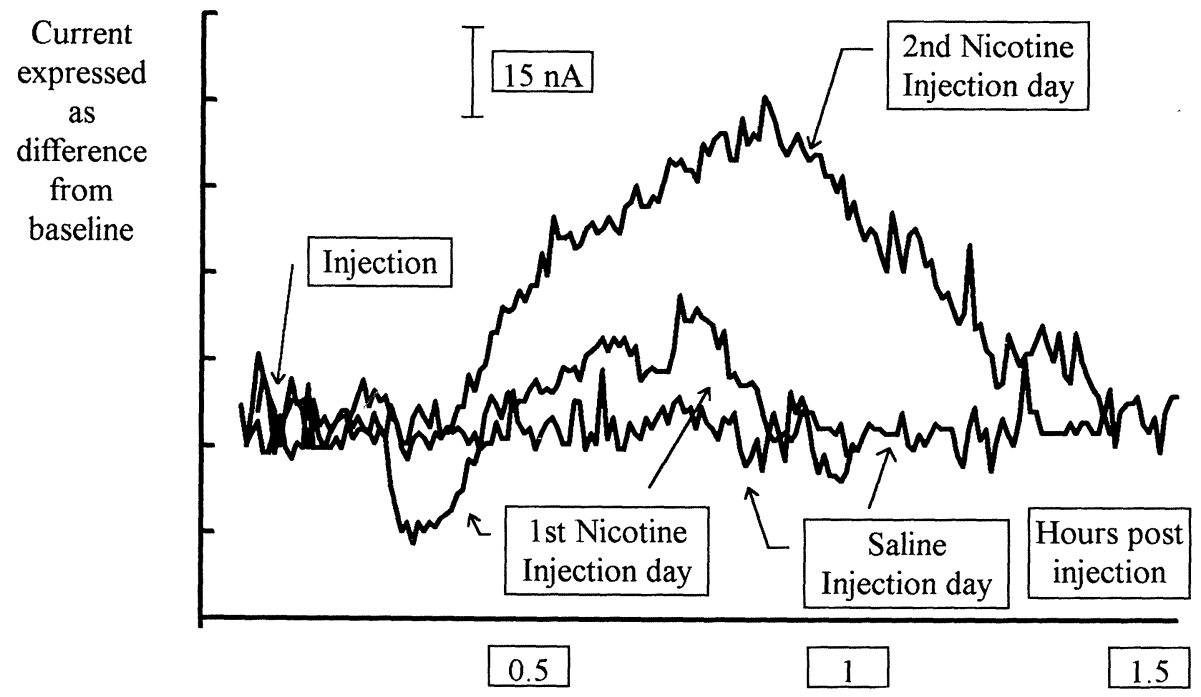

Figure 5. Response at Accumbal Nafion-modified electrodes to systemic injections of saline or nicotine $(0.4 \mathrm{mg} / \mathrm{kg} \mathrm{SC}$ calculated as base). Data shows response to saline and to injections of nicotine on three separate days $(\mathrm{n}=4$ rats). 
an increase lasting approximately $1 \mathrm{hr}$. On the second nicotine day, there was no decrease in signal and a much larger increase that was maximal at $45 \mathrm{~min}$ and returned to baseline 90 min after injection.

\section{Discussion}

The high temporal resolution afforded by electrochemistry has allowed us to capture a rapid and transient decrease in mesolimbic DA levels following systemic administration of nicotine to nicotine-naive rats. This decrease is an interesting finding of these preliminary data. That the response on the second injection day should be a more rapid increase to a greater maximum finds parallels with the behavioural responses to systemic nicotine and is consistent with observations made using microdialysis (4).

Electrochemistry clearly offers a useful tool for monitoring transmitter levels in drug and behavioural tests. However, there are certain caveats to its use. Obviously it can only measure levels of substances that are electroactive. Furthermore, it does not have the neurochemical discrimination of microdialysis. The careful verification of such a technique is vital before it can usefully be used to answer neurobiological questions.

Acknowledgements: This work was supported by a grant from the National Science and Engineering Research Council of Canada.

\section{References}

1. Corrigall WA, Franklin KJB, Coen KM, Clarke PBS. The mesolimbic dopaminergic system is implicated in the reinforcing effects of nicotine. Psychopharm 1992; 107: 285-289.

2. Stolerman IP, Shoaib M. The neurobiology of tobacco addiction. TiPS 1991; 12: 467-473.

3. Clarke PBS. Dopaminergic mechanisms in the locomotor stimulant effects of nicotine. Biochem Pharm 1990; 40: 1427-1432.

4. Benwell MEM, Balfour DJK. The effects of acute and repeated nicotine treatment on nucleus accumbens dopamine and locomotor activity. Br J Pharmacol 1992; 105: 849-856.

5. Gerhardt GA, Oke AF, Nagy G, Moghaddam B, Adams RN. Nafion-coated electrodes with high selectivity for CNS electrochemistry. Brain Res 1984; 290: 390-395.

6. Lane RF, Blaha CD, Hari SP. Electrochemistry in vivo: monitoring dopamine release in the brain of the conscious, freely moving rat. Brain Res Bull 1987; 19: 19-27.

7. Kruk ZL J Neurosci Meth (submitted).

8. Zetterstrom T, Wheeler DB, Boutelle MG, Fillenz M. Striatal ascorbate and its relationship to dopamine receptor stimulation and motor activity. Eur J Neurosci 1991; 3: 940-946. 